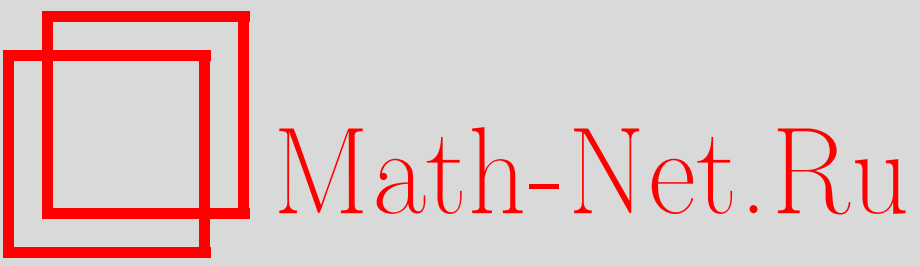

Ю. Г. Рыков, Вариационный принцип для двумерной системы уравнений газовой динамики без давления, УМН, 1996, том 51, выпуск 1, 165-166

DOI: https://doi.org/10.4213/rm935

Использование Общероссийского математического портала Math-Net.Ru подразумевает, что вы прочитали и согласны с пользовательским соглашением

http://www.mathnet.ru/rus/agreement

Параметры загрузки:

IP : 35.173 .137 .237

26 апреля 2023 г., 14:29:01 


\title{
ВАРИАЦИОННЫЙ ПРИНЦИП ДЛЯ ДВУМЕРНОЙ СИСТЕМЫ УРАВНЕНИЙ ГАЗОВОЙ ДИНАМИКИ БЕЗ ДАВЛЕНИЯ
}

\author{
Ю. Г. Рыков
}

Рассмотрена следующая система уравнений

$$
\left\{\begin{array}{l}
\partial \varrho / \partial t+\partial\left(\varrho u_{1}\right) / \partial x_{1}+\partial\left(\varrho u_{2}\right) / \partial x_{2}=0, \\
\partial\left(\varrho u_{1}\right) / \partial t+\partial\left(\varrho u_{1}^{2}\right) / \partial x_{1}+\partial\left(\varrho u_{1} u_{2}\right) / \partial x_{2}=0, \\
\partial\left(\varrho u_{2}\right) / \partial t+\partial\left(\varrho u_{1} u_{2}\right) / \partial x_{1}+\partial\left(\varrho u_{2}^{2}\right) / \partial x_{2}=0,
\end{array} \quad\left(t, x_{1}, x_{2}\right) \in R_{+} \times R^{2},\right.
$$

где $\varrho$ - плотность,$\left(u_{1}, u_{2}\right)$ - вектор скорости; с начальными данными

$$
\begin{aligned}
\varrho\left(0, x_{1}, x_{2}\right) & =\varrho_{0}\left(x_{1}, x_{2}\right)>0 \in C\left(R^{2}\right), \\
u_{1}\left(0, x_{1}, x_{2}\right) & =u_{1}^{(0)}\left(x_{1}, x_{2}\right) \in C\left(R^{2}\right) \\
u_{2}\left(0, x_{1}, x_{2}\right) & =u_{2}^{(0)}\left(x_{1}, x_{2}\right) \in C\left(R^{2}\right) .
\end{aligned}
$$

Пусть существуют такие непрерывно-дифференцируемые функции $\left(A\left(x_{1}, x_{2}\right), B\left(x_{1}, x_{2}\right)\right): R^{2}=$ $\left(x_{1}, x_{2}\right) \rightarrow G \subset R^{2}=(A, B)$, что

$$
\begin{gathered}
\partial(A, B) / \partial\left(x_{1}, x_{2}\right)=\varrho_{0}\left(x_{1}, x_{2}\right)>0 ; \quad \partial x_{1} / \partial B=\partial x_{2} / \partial A \\
\partial u_{1}^{(0)}\left(x_{1}(A, B), x_{2}(A, B)\right) / \partial B=\partial u_{2}^{(0)}\left(x_{1}(A, B), x_{2}(A, B)\right) / \partial A .
\end{gathered}
$$

Обозначим через $S_{0}(A, B)$ соответствующий потенциал, т.е.

$$
\partial S_{0} / \partial A=u_{1}^{(0)}\left(x_{1}(A, B), x_{2}(A, B)\right), \quad \partial S_{0} / \partial B=u_{2}^{(0)}\left(x_{1}(A, B), x_{2}(A, B)\right) .
$$

Рассмотрим также выпуклую функцию $\Phi(A, B)$ такую, что

$$
\partial \Phi / \partial A=x_{1}(A, B) ; \quad \partial \Phi / \partial B=x_{2}(A, B) .
$$

Эта функция существует ввиду (3). Пусть выполнены условия

$$
\begin{aligned}
& \Phi(A, B) \rightarrow+\infty \text { при }(A, B) \rightarrow \partial G, \\
& \sup _{A^{2}+B^{2}=R^{2}} \frac{\left|S_{0}(A, B)\right|}{\Phi(A, B)} \rightarrow 0 \text { при } 2 R \rightarrow \operatorname{diam} G .
\end{aligned}
$$

Естественно считать, что (1) задает динамику в пространстве троек $\left(\mathrm{P}_{t},\left(I_{1}\right)_{t},\left(I_{2}\right)_{t}\right)$, где $\mathrm{P}_{t}$ есть мера, отвечающая распределению масс, а $\left(I_{1}\right)_{t},\left(I_{2}\right)_{t}$ - меры Радона, отвечающие распределению импульса, в момент времени $t$. При этом $\left(I_{1}\right)_{t},\left(I_{2}\right)_{t}$ - абсолютно непрерьвны относительно $\mathrm{P}_{t}$ и производные Радона-Никодима $d\left(I_{1}\right)_{t}\left(x_{1}, x_{2}\right) / d \mathrm{P}_{t}\left(x_{1}, x_{2}\right)=u_{1}\left(t, x_{1}, x_{2}\right)$, $d\left(I_{2}\right)_{t}\left(x_{1}, x_{2}\right) / d \mathrm{P}_{t}\left(x_{1}, x_{2}\right)=u_{2}\left(t, x_{1}, x_{2}\right)$, есть вектор скорости в точке $\left(x_{1}, x_{2}\right)$. Пусть $f, g, h \in$ $C_{0}^{1}\left(R^{2}\right)$.

ОПредЕлЕниЕ 1 . Семейство $\left.\left(\mathrm{P}_{t},\left(I_{1}\right)_{t},\left(I_{2}\right)_{t}\right)\right), 0 \leqslant t \leqslant T$, назовем слабым решением задачи (1), (2) на отрезке [0,T], если для любых $0 \leqslant t_{1}<t_{2} \leqslant T$

$$
\begin{aligned}
& \iint f\left(x_{1}, x_{2}\right) d \mathrm{P}_{t_{2}}\left(x_{1}, x_{2}\right)-\iint f\left(x_{1}, x_{2}\right) d \mathrm{P}_{t_{1}}\left(x_{1}, x_{2}\right) \\
& =\int_{t_{1}}^{t_{2}}\left\{\iint \frac{\partial f}{\partial x_{1}}\left(x_{1}, x_{2}\right) d\left(I_{1}\right)_{\tau}\left(x_{1}, x_{2}\right)+\iint \frac{\partial f}{\partial x_{2}}\left(x_{1}, x_{2}\right) d\left(I_{2}\right)_{\tau}\left(x_{1}, x_{2}\right)\right\} d \tau \\
& \iint g\left(x_{1}, x_{2}\right) d\left(I_{1}\right)_{t_{2}}\left(x_{1}, x_{2}\right)-\iint g\left(x_{1}, x_{2}\right) d\left(I_{1}\right)_{t_{1}}\left(x_{1}, x_{2}\right)
\end{aligned}
$$

Работа выполнена при финансовой поддержке Российского фонда фундаментальных исследований (грант № 93-01-16090). 


$$
\begin{array}{r}
=\int_{t_{1}}^{t_{2}}\left\{\iint \frac{\partial g}{\partial x_{1}}\left(x_{1}, x_{2}\right) u_{1}\left(\tau, x_{1}, x_{2}\right) d\left(I_{1}\right)_{\tau}\left(x_{1}, x_{2}\right)\right. \\
\left.+\iint \frac{\partial g}{\partial x_{2}}\left(x_{1}, x_{2}\right) u_{1}\left(\tau, x_{1}, x_{2}\right) d\left(I_{2}\right)_{\tau}\left(x_{1}, x_{2}\right)\right\} d \tau \\
\iint h\left(x_{1}, x_{2}\right) d\left(I_{2}\right)_{t_{2}}\left(x_{1}, x_{2}\right)-\iint h\left(x_{1}, x_{2}\right) d\left(I_{2}\right)_{t_{1}}\left(x_{1}, x_{2}\right) \\
=\int_{t_{1}}^{t_{2}}\left\{\iint \frac{\partial h}{\partial x_{1}}\left(x_{1}, x_{2}\right) u_{2}\left(\tau, x_{1}, x_{2}\right) d\left(I_{1}\right)_{\tau}\left(x_{1}, x_{2}\right)\right. \\
\left.+\iint \frac{\partial h}{\partial x_{2}}\left(x_{1}, x_{2}\right) u_{2}\left(\tau, x_{1}, x_{2}\right) d\left(I_{2}\right)_{\tau}\left(x_{1}, x_{2}\right)\right\} d \tau
\end{array}
$$

и $\mathrm{P}_{t} \rightarrow \mathrm{P}_{0},\left(I_{1}\right)_{t} \rightarrow I_{1}^{(0)},\left(I_{2}\right)_{t} \rightarrow I_{2}^{(0)}$ при $t \rightarrow+0$ в слабой топологии на любом компакте $R^{2}$, где $\mathrm{P}_{0}, I_{1}^{(0)}, I_{2}^{(0)}$ определяются начальными функциями $(2)$.

Следуя Зельдовичу [1]-[3], мы рассматриваем динамику свободно движущихся частиц, которые взаимодействуют посредством столкновений в соответствии с законами сохранения массы и импульса. В отличие от [2], [3] при фиксированном $t \in[0, T]$ для всякой точки с координатами $\left(x_{1}, x_{2}\right)$ нельзя указать частиц, пришедших в эту точку к моменту времени $t$, а можно указать лишь соответствующие распределения массы и импульса. Поскольку характеристики системы (1) известны, решение $\left(\mathrm{P}_{t},\left(I_{1}\right)_{t},\left(I_{2}\right)_{t}\right)$ будет однозначно определено, если для каждого $t \in[0, T]$ мы укажем разбиение $\xi_{t}$ плоскости $R^{2}$, элементы $C_{\xi_{t}}$ которого представляют собой связные выпуклые подмножества $R^{2}$, определяющие искомые меры. Для построения элементов $C_{\xi_{t}}$ для каждого $t \in[0, T]$ рассмотрим выпуклую оболочку $C_{\Omega}(t)$ функции $\Omega(t, A, B) \equiv \Phi(A, B)+t S_{0}(A, B)$. Тогда $C_{\Omega}(t)$ порождает разбиение $\xi_{t}$ плоскости $(A, B)$. Элементами разбиения $\xi_{t}$ служат множества $p_{t}\left(x_{1}, x_{2}\right)$, на которых $C_{\Omega}(t)$ совпадает с подходящей линейной функцией $x_{1} A+x_{2} B+z\left(x_{1}, x_{2}\right)$.

Теперь обсудим получающееся обобщение условий Ренкина-Гюгонио для (1), выражающее собой закон сохранения импульса. Пусть $p_{t}\left(x_{1}, x_{2}\right)$ является треугольником с вершинами $\left(A_{1}, B_{1}\right) ;\left(A_{2}, B_{2}\right) ;\left(A_{3}, B_{3}\right)$. Рассмотрим треугольник $T$ в пространстве $(S, A, B)$ с вершинами $\left(S_{1}, A_{1}, B_{1}\right) ;\left(S_{2}, A_{2}, B_{2}\right) ;\left(S_{3}, A_{3}, B_{3}\right)$, где $S_{j} \equiv S_{0}\left(A_{j}, B_{j}\right)(j=1,2,3), S_{0}$ определяется формулой (5). Оказывается, что точка $X=\left(x_{1}, x_{2}\right)$ будет обладать массой $\mathrm{P}(X)$, равной площади проекции $T$ на плоскость $S=0$ и вектор-импульсом $\left(I_{1}(X), I_{2}(X)\right)$ таким, что $I_{1}(X)$ равно ориентированной площади проекции $T$ на плоскость $A=0, \mathrm{a}-I_{2}(X)$ равно ориентированной площади проекции $T$ на плоскость $B=0$.

Пусть теперь $p_{t}\left(x_{1}(l), x_{2}(l)\right)$ является отрезком с концами $\left(A_{1}(l), B_{1}(l)\right),\left(A_{2}(l), B_{2}(l)\right)$ для точек $\Gamma=\left(x_{1}(l), x_{2}(l)\right)$, лежащих на некоторой кривой, $l$ - параметр вдоль этой кривой. В пространстве $(S, A, B)$ рассмотрим кривые $\left(S_{1}(l), A_{1}(l), B_{1}(l)\right) ;\left(S_{2}(l), A_{2}(l), B_{2}(l)\right)$, где $S_{j}(l) \equiv S_{0}\left(A_{j}(l), B_{j}(l)\right)(j=1,2), S_{0}$ определяется формулой $(5)$. Для каждого значения $l$ рассмотрим бесконечно малый четырехугольник П $(l)$ с вершинами $\left(S_{1}, A_{1}, B_{1}\right) ;\left(S_{2}, A_{2}, B_{2}\right)$; $\left(S_{2}+\frac{d S_{2}}{d l} \Delta l, A_{2}+\frac{d A_{2}}{d l} \Delta l, B_{2}+\frac{d B_{2}}{d l} \Delta l\right) ;\left(S_{1}+\frac{d S_{1}}{d l} \Delta l, A_{1}+\frac{d A_{1}}{d l} \Delta l, B_{1}+\frac{d B_{1}}{d l} \Delta l\right)$. Тогда бесконечно малый участок $\Delta \Gamma$ кривой $\Gamma$ будет обладать массой $\mathrm{P}(\Delta \Gamma)$, равной площади проекции П на плоскость $S=0$ и вектор-импульсом $\left(I_{1}(\Delta \Gamma), I_{2}(\Delta \Gamma)\right)$ таким, что $I_{1}(\Delta \Gamma)$ равно ориентированной площади проекции П на плоскость $A=0$, a $-I_{2}(\Delta \Gamma)$ равно ориентированной площади проекции П на плоскость $B=0$.

Теорема 1. Пусть выполнены условия (3), (4), (7). Тогда для всякого $T>0$ построенное семейство разбиений $\xi_{t}$ порождает слабое решение задачи (1), (2).

\section{СПИСОК ЛИТЕРАТУРЫ}

[1] Zeldovich Ya. В. // Astron. Astrophys. 1970. V. 5. Р. 84-89. [2] Вейнан И., Рыков Ю. Г., Синай Я. Г. // УМН. 1995. Т. 50. С. 193-194. [3] Weinan E., Rykov Yu. G., Sinai Ya. G. // Comm. Math. Phys. 1995 (to appear). 